\title{
Description of beam instabilities in synchrotrons with wakefields and space charge forces using the circulant matrix model
}

\author{
X. Buffat ${ }^{*}$ and E. Métral@ \\ European Organization for Nuclear Research (CERN), CH-1211 Geneva, Switzerland \\ E. Gottlob® \\ École Polytechnique Fédérale de Lausanne (EPFL), CH-1015 Lausanne, Switzerland \\ and Ecole Polytechnique de Bruxelles, BE-1050 Bruxelles, Belgium \\ C. $\mathrm{Høgh} \odot$ \\ École Polytechnique Fédérale de Lausanne (EPFL), CH-1015 Lausanne, Switzerland \\ and Imperial College, London SW7 2BU, United Kingdom \\ A. Oeftiger \\ European Organization for Nuclear Research (CERN), CH-1211 Geneva, Switzerland \\ and GSI Helmholtzzentrum für Schwerionenforschung, Planckstraße 1, 64291 Darmstadt, Germany \\ T. Pieloni \\ École Polytechnique Fédérale de Lausanne (EPFL), CH-1015 Lausanne, Switzerland
}

(Received 24 March 2021; accepted 14 June 2021; published 25 June 2021)

\begin{abstract}
A new model for the description of beam instabilities in synchrotrons featuring wakefields and space charge forces is proposed, using the circulant matrix approach. The predictions of this model are discussed in light of past ones, with a particular emphasis on the possible mitigation of the transverse mode coupling instability by space charge forces. The existence of transient amplification in spite of the absence of unstable eigenvalues in configuration featuring strong space charge forces is also addressed. It is shown that this behavior can be recovered when considering an airbag distribution. Yet when considering a more realistic Gaussian distribution, the radial modes lead to other types of mode coupling instabilities. The predictions of the new model are then compared to results of an experiment conducted at the CERN Super Proton Synchrotron, showing a reasonable agreement.
\end{abstract}

DOI: 10.1103/PhysRevAccelBeams.24.060101

\section{INTRODUCTION}

An accurate description of the beam instabilities in the presence of space charge forces and other types of wakefields is rather difficult to obtain in the general case. By approximating the beam distribution with an airbag and the longitudinal potential with a square well (ABS), a description that remains valid for arbitrarily strong space charge was found [1]. This description predicts that the transverse mode coupling instability (TMCI) threshold increases for increasing space charge forces. This prediction was also confirmed considering linear synchrotron motion as well as

\footnotetext{
*xavier.buffat@cern.ch

Published by the American Physical Society under the terms of the Creative Commons Attribution 4.0 International license. Further distribution of this work must maintain attribution to the author(s) and the published article's title, journal citation, and DOI.
}

radial modes, yet maintaining a boxcar longitudinal distribution [2]. The absence of TMCI was also obtained within the strong space charge approximation (SSC) [3-5]. With the latter, it is shown that even in the absence of normal instabilities, i.e., characterized by an unstable eigenvalue, instabilities of convective type remain [6]. All these predictions appear in contradiction to the two particles approach (2PA) which shows that when increasing the space charge strength, the original TMCI is indeed suppressed, however it is replaced by a succession of unstable and stable conditions, in the normal sense, i.e., characterized by unstable eigenvalues [7], as reported in Fig. 1. In order to improve both the understanding of the observed instability mechanisms, as observed for example at CERN's Super Proton Synchrotron (SPS) [8], and the accuracy of the beam stability predictions, we propose another semianalytical approach based on the circulant matrix model (CMM) [9]. The classical analysis of the unstable eigenvalues of the matrix will be complemented 


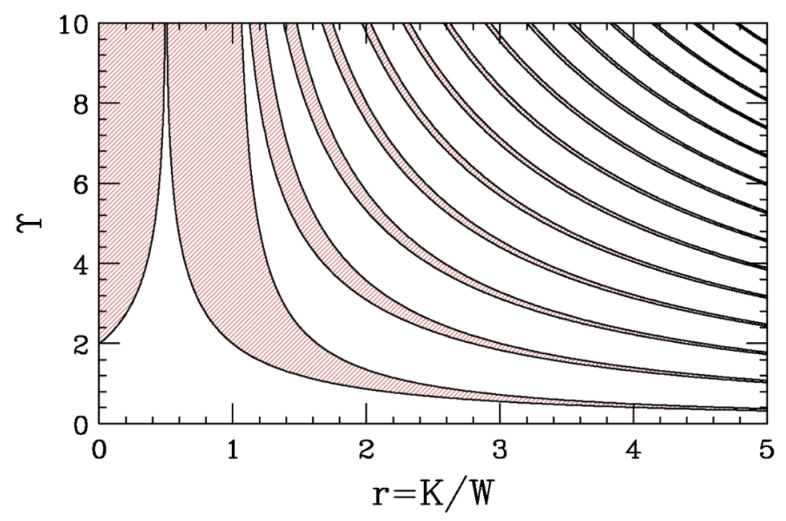

FIG. 1. Beam stability obtained with the 2PA as a function of the wakefield strength represented by $\Upsilon$ and $W$, and the space charge strength represented by $K$. The red areas mark configurations featuring unstable eigenvalues. This plot was taken from [7].

by a characterization of transient amplification mechanisms, making use of the Kreiss constant theorem [10], allowing us to bridge to the convective behavior recently obtained within the SSC.

In Sec. II, we describe the analytical and the numerical algorithm to obtain the one turn matrix including linearized space charge forces and wakefields for an airbag and a Gaussian distribution. In Sec. III, we recover the results obtained within the ABS and SSC, i.e., the apparent mitigation of the TMCI by space charge forces and the transient amplification that may occur, even in configurations featuring no unstable eigenvalues. In Sec. IV, we show that the unstable conditions predicted by the $2 \mathrm{PA}$ are recovered in the form of transverse mode couplings and decouplings of various radial modes. Finally, in Sec. V we compare qualitatively our model's prediction to past measurements at the SPS, before concluding in Sec. VI. The numerical convergence of the new model as well as a concrete example of transient amplification are discussed in the Appendixes.

For simplicity, we consider in the following a broadband resonator model for the wakefields [11], defined by a resonant frequency $f_{r}$, a shunt impedance $R_{r}$ and a quality factor $Q_{r}$. Following [12], we define the normalized intensity:

$$
I_{\text {norm }}=\frac{q^{2} N_{b} \beta_{\mathrm{avg}}}{2 \gamma_{r} m c^{2} Q_{s}} \frac{f_{r} R_{r}}{Q_{r}}
$$

with $q$ and $m$ the particle's electric charge and mass, $N_{b}$ the number of particles in the bunch, $\beta_{\text {avg }}$ the average optical function around the ring, $\gamma_{r}$ the relativistic factor, $c$ the speed of light and $Q_{s}$ the synchrotron tune. For improved readability we also restrict ourselves to a bunch length that follows $f_{r} \tau_{b}=1$ and a quality factor $Q_{r}=1$, which is rather close to the constant wake approach often used in literature. Nevertheless, our approach is not limited to this assumption, as shown for example in [13].

\section{SPACE CHARGE WITHIN THE CIRCULANT MATRIX MODEL}

The CMM was first introduced to assess the suppression of the transverse mode coupling instability (TMCI) with a transverse feedback [9]. It turns out to be a rather versatile model, capable of consistently describing the impact of the transverse feedback with various other coherent forces such as the beam-beam interactions or driving and detuning wakefields [13-15]. The CMM has also successfully been employed to understand the impact of second order chromaticity on the head-tail instability [16]. These effects are implemented in a code called BIMBIM [17]. The implementation of a model of space charge forces in this code therefore offers the potential for studying the interplay with different effects.

Let us briefly recall the concept of the CMM. We start with a polar discretization of the normalized longitudinal phase space, as depicted on Fig. 2. The particles' coordinates are therefore related to the longitudinal position $z$ and the relative momentum deviation $\delta$ with

$$
\begin{gathered}
r^{2}=\left(\frac{z}{\sigma_{z}}\right)^{2}+\left(\frac{\delta}{\sigma_{\delta}}\right)^{2}, \\
\tan (\theta)=\frac{\delta}{z},
\end{gathered}
$$

with $\sigma_{z}$ and $\sigma_{\delta}$ the rms bunch length and relative momentum spread respectively. In the azimuthal direction the so-called slices are delimited by their boundaries

$$
\Theta_{i}=2 \pi \frac{i+0.5}{N_{s}}, \quad i=0, \ldots, N_{s},
$$

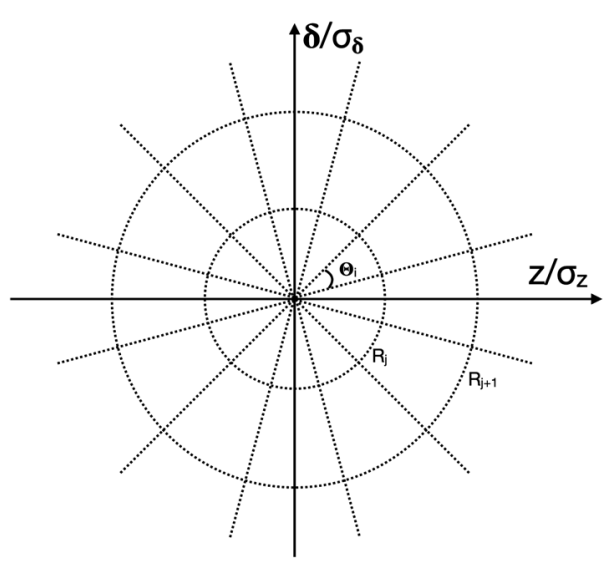

FIG. 2. Illustration of the discretization of the longitudinal phase space in slices and rings within the CMM. 
with $N_{s}$ the number of slices. For an airbag distribution, no discretization is required in the radial direction. When considering a Gaussian distribution, we chose a uniform discretization truncating the distribution at $N_{t} \sigma_{z}$, the boundaries are therefore given by

$$
R_{j}=N_{t} \frac{j}{N_{r}}, \quad j=0, \ldots, N_{r},
$$

with $N_{r}$ the number of rings. In the following, we shall use a truncation at $3 \sigma_{z}$. In order to describe the behavior of the beam, we seek to obtain the one turn matrix for the centroid motion of each discretized element, or beamlet, in the transverse plane. The centroid motion is characterized by its average position with respect to the close orbit $x_{k}$ and average transverse momentum normalized to the total momentum $x_{k}^{\prime}$. In the following, we shall only consider a single transverse plane, but the method is in principle applicable to more degrees of freedom. The synchrobetatron motion in the lattice is then described by a matrix $M_{l}$ given by the Kronecker product of a rotation matrix with tune $Q_{\beta}, \mathcal{R}\left(Q_{\beta}\right)$, corresponding to the transverse motion of the beamlets and a circulant matrix representing the rotation within each ring due to the longitudinal focusing. The latter is obtained with the Kronecker product of the identity matrix with a size given by the number of rings $\mathbb{I}_{N_{r}}$ and a permutation matrix with a size given by the number of slices $P_{N_{s}}$ exponentiated by the number of slices times the synchrotron tune $Q_{s}$ [9]:

$$
M_{l}\left(Q_{\beta}, Q_{s}\right)=\mathbb{I}_{N_{r}} \otimes P_{N_{s}}^{N_{s} Q_{s}} \otimes \mathcal{R}\left(Q_{\beta}\right) .
$$

The implementation of space charge forces in this framework requires the description of the interaction between the beamlets. We start by writing the variation of the divergence $x_{t}^{\prime}$, or kick, of a test particle at the transverse and longitudinal positions $x_{t}$ and $z_{t}$ respectively, under the influence of the electric field caused by a source beam with average position $\langle x\rangle$ [18]. We use the highly relativistic approximation such that electromagnetic fields can be considered as purely transverse. In addition we use the smooth approximation, i.e., constant optics functions around the machine of circumference $L$ :

$$
\Delta x_{t}^{\prime}=\frac{q^{2} N_{b} \lambda_{s}\left(z_{t}\right) L}{4 \pi \epsilon_{0} m c^{2} \beta_{r}^{2} \gamma_{r}^{3} \beta_{\text {avg }} \epsilon_{t}}\left(x_{t}-\langle x\rangle\right) \hat{\delta}\left(z_{t}\right),
$$

with $\epsilon_{0}$ the vacuum permittivity, $\beta_{r}$ the relativistic velocity factor and $\hat{\delta}(\cdot)$ the Dirac delta function. The physical transverse emittance of the beam is given by $\epsilon_{t} \cdot \lambda_{s}\left(z_{t}\right)$ is the normalized line density at the location of the test particle. Generically, we define the normalized particle distribution of the source beamlet $\Psi_{s}(z, \delta)$ such that we can compute the kick of beamlet $s$ on the test particle $t$ :

$$
\Delta x_{t, s}^{\prime}=A \sigma_{z}\left(x_{t}-\langle x\rangle_{s}\right) \int d \delta \Psi_{s}\left(z_{t}, \delta\right)
$$

with

$$
A=\frac{q^{2} N_{b} L}{4 \pi \epsilon_{0} m c^{2} \beta_{r}^{2} \gamma_{r}^{3} \beta_{\mathrm{avg}} \epsilon_{t} \sigma_{z}} .
$$

We now conveniently replace the distribution of the beamlet at the location of the test particle with a Dirac delta function:

$$
\Psi_{s}\left(z_{t}, \delta\right) \rightarrow \int d z \Psi_{s}(z, \delta) \hat{\delta}\left(z-z_{t}\right)
$$

and we integrate the kick of an entire test beamlet with distribution $\Psi_{t}(z, \delta)$ :

$$
\begin{aligned}
\left\langle\Delta x_{t, s}^{\prime}\right\rangle= & A \sigma_{z}\left(\langle x\rangle_{t}-\langle x\rangle_{s}\right) \\
& \times \frac{\iiint \int d z_{s} d \delta_{s} d z_{t} d \delta_{t} \Psi_{s}\left(z_{s}, \delta_{s}\right) \Psi_{t}\left(z_{t}, \delta_{t}\right) \hat{\delta}\left(z_{s}-z_{t}\right)}{\iint d z_{t} d \delta_{t} \Psi_{t}\left(z_{t}, \delta_{t}\right)} .
\end{aligned}
$$

This integral can then be expressed in the normalized polar coordinate system written above, defining the distribution of the beamlets in polar coordinates and simplifying the notation $\Psi_{k}^{p}\left(r_{k}, \theta_{k}\right) \equiv \Psi_{k}^{p}$ :

$$
\begin{aligned}
\left\langle\Delta x_{t, s}^{\prime}\right\rangle= & A\left(\langle x\rangle_{t}-\langle x\rangle_{s}\right) \\
& \times \frac{\iiint \int d r_{s} d \theta_{s} d r_{t} d \theta_{t} \Psi_{s}^{p} \Psi_{t}^{p} \hat{\delta}\left[r_{s} \sin \left(\theta_{s}\right)-r_{r} \sin \left(\theta_{r}\right)\right]}{\iint d r_{t} d \theta_{t} \Psi_{t}^{p}} .
\end{aligned}
$$

Based on this expression one can write a space charge matrix describing the change of the transverse coordinates of each beamlet based on their coordinates at the previous turn:

$$
x_{k} \rightarrow x_{k}, \quad x_{k}^{\prime} \rightarrow x_{k}^{\prime}+\sum_{s}\left\langle\Delta x_{k, s}^{\prime}\right\rangle .
$$

The full one turn matrix including the phase space rotation through the lattice and space charge forces is obtained with an outer product of the matrix $C\left(Q_{s}\right)$ and the space charge matrix defined by Eq. (13). In the following, we compute the space charge matrix for two different distributions and study the properties of the dynamical system notably via the eigenvalues of the one turn matrix. The eigenvalues are expressed in terms of a tune shift with respect to the unperturbed tune $Q_{\beta}$. By convention, a positive imaginary part corresponds to an exponentially growing amplitude. 
For convenience, we characterize the strength of the space charge forces with the maximum induced tune shift for a Gaussian beam in the smooth and round beam approximation. It can be expressed based on Eq. (12):

$$
\Delta Q_{\mathrm{SC}}=A \frac{\beta_{\mathrm{avg}}}{4 \pi} \frac{1}{\sqrt{2 \pi}} .
$$

\section{A. Airbag distribution}

The airbag distribution is often used to describe coherent instabilities due to its simplicity and consequently its value for the understanding of the instability mechanisms. We define the discretized distributions as

$$
\Psi_{A B, i}^{p}(r, \theta)= \begin{cases}\frac{N_{s}}{2 \pi} \hat{\delta}\left(r-R_{0}\right), & \text { if } \theta \in\left[\Theta_{i}, \Theta_{i+1}[\right. \\ 0, & \text { otherwise. }\end{cases}
$$

In order to obtain the equivalent rms bunch length and momentum spread, the radius of the airbag should be set to $R_{0}=\sqrt{2}$. With a substitution we can solve the corresponding integral in Eq. (12):

$$
\left\langle\Delta x_{t, s}^{\prime}\right\rangle=\left\{\begin{array}{l}
\frac{A}{8 \pi^{2} R_{0}}\left(\langle x\rangle_{t}-\langle x\rangle_{s}\right)\left|\log \left(\frac{1+U_{s+1}}{1-U_{s+1}} \frac{1-U_{s}}{1+U_{s}}\right)\right|, \quad \text { if } t+s=N_{s} \text { and } t \neq s \\
0, \quad \text { otherwise }
\end{array}\right.
$$

with the new integration boundaries $U_{i}=\cos \Theta_{i}$.

\section{B. Gaussian distribution}

The simplicity of the airbag distribution is not without cost as it prevents the description of modes with a radial component. In order to address those modes, we instead consider a discretized Gaussian distribution in normalized polar coordinates:

$$
\Psi_{G, i, j}^{p}(r, \theta)= \begin{cases}\frac{r}{2 \pi} e^{-\frac{r^{2}}{2}}, & \text { if } r \in\left[R_{i}, R_{i+1}[\right. \\ & \text { and } \theta \in\left[\Theta_{i}, \Theta_{i+1}[\right. \\ 0, & \text { otherwise. }\end{cases}
$$

The integral in Eq. (12) can then be solved numerically. Here we replaced the Dirac delta function by a boxcar function of vanishing width and used a trapezoidal scheme for the integration.

\section{AIRBAG DISTRIBUTION}

The variation of the coherent mode frequencies due to space charge in the absence of wakefields is shown in Fig. 3(a). In the absence of space charge forces, we find as expected a series of modes equally spaced by integer multiples of the synchrotron tune $Q_{s}$ around the transverse tune $Q_{\beta}$, corresponding to the azimuthal modes of oscillation. Consistently with the ABS model, we find that the frequency of the azimuthal mode 0 is fixed at the unperturbed transverse tune, while the frequency of all others is decreased, with a saturation at the unperturbed tune for the positive azimuthal modes [1]. The modes' frequencies are slightly different from the ones obtained with the ABS, which can be attributed to the difference in longitudinal focusing between the two approaches.
When considering wakefields at fixed strength, yet varying the strength of space charge forces, we observe in Fig. 3(b) that the original TMCI is suppressed. Indeed,

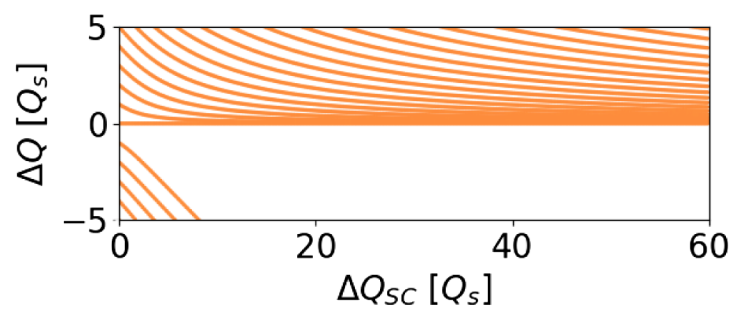

(a)

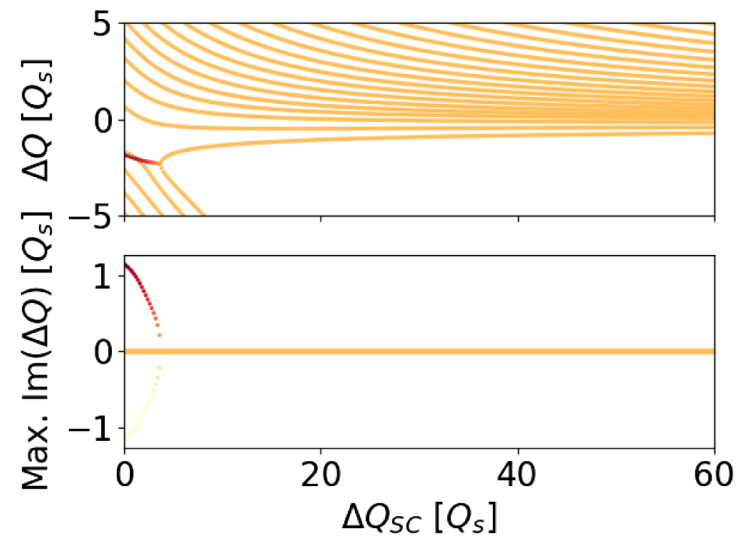

(b)

FIG. 3. Evolution of the complex tune shifts of the coherent modes as a function of the normalized space charge tune shift for an airbag distribution, without wakefields (above) and with wakefields featuring fixed wakefield strength corresponding to a normalized intensity of 20 (below). In the absence of wakefields, all tunes are purely real. Otherwise, to ease the identification of the modes in the plots of the real and imaginary tune shifts, the dots are color coded identically according to the imaginary part. (a) Without wake elds (b) With wakefields. 
observing the imaginary part of the most unstable mode we find that it decreases with increasing space charge strength until reaching stabilization at a space charge tune shift of approximately 5 . The mechanism of stabilization can be understood by observing the corresponding real tune shift (red dots), starting approximately at $-2 Q_{s}$ without space charge and decoupling back into two stable modes. The frequency of one of the formerly coupled mode is shifted up towards the positive azimuthal modes, while the other's is shifted down towards negative azimuthal modes.

The suppression of the TMCI when increasing the space charge strength obtained with the CMM based on an airbag distribution is compatible with the findings of the ABS model. The dependence of the imaginary part of the most unstable mode on both the strength of the space charge forces and of the wakefields is detailed in Fig. 4(a). The unstable area for low space charge forces is comparable to the one obtained with the 2PA (Fig. 1). On the other hand, all the other unstable areas obtained with the 2PA for high

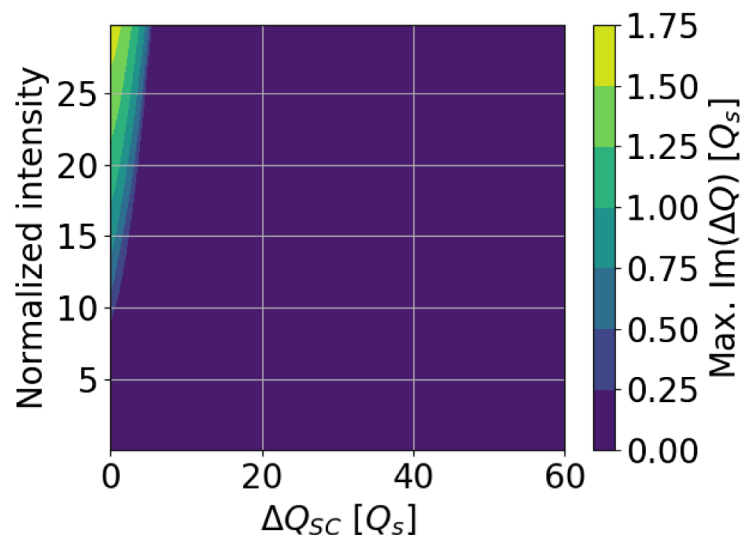

(a)

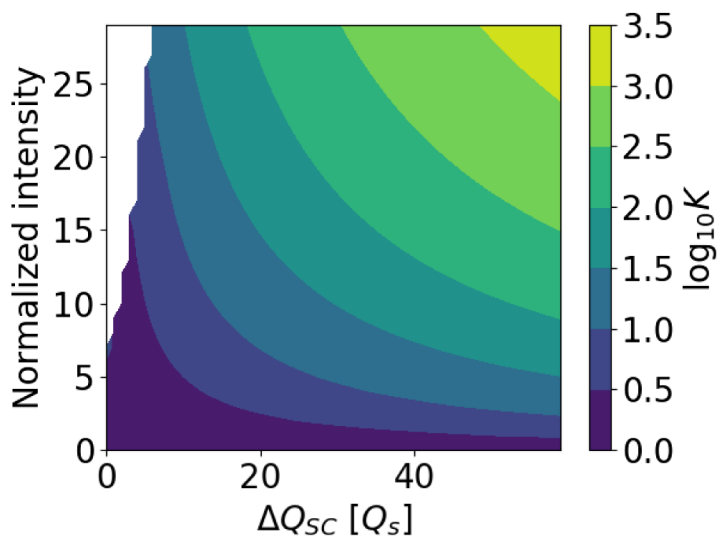

(b)

FIG. 4. Maximum tune shift (above) and the Kreiss constant (below) obtained for an airbag distribution for various strength of space charge forces and wakefields. The Kreiss constant becomes infinite for configurations featuring unstable eigenvalues which is marked by a white area. (a) Maximum tune shift (b) Kreiss constant. space charge strength are not recovered with the CMM. As discussed later, this inconsistency is related to a description of the radial degree of freedom, which is lacking in both the CMM with an airbag distribution and the ABS. Nevertheless the total absence of unstable behavior remains rather surprising, given that even a much simpler model such as the 2PA seems to yield a better description of the instabilities. We therefore inspect the sensitivity of the matrix to external perturbations using the so-called pseudospectrum of the one turn matrix $M$, defined as [19]

$$
\sigma_{p}\left(M, \epsilon_{p}\right)=\left\{\lambda \in \mathbb{C} \mid \exists \vec{v}:\|(M-\lambda I) \cdot \vec{v}\|<\epsilon_{p}\right\} .
$$

As it yields the eigenvalues that can be obtained by adding a perturbation of order $\epsilon_{p}$ to the matrix, the extension of the pseudospectrum into the imaginary plane provides a measure of the sensitivity of a dynamical system to perturbation. Figure 5 shows with contours the pseudospectrum corresponding to different perturbation amplitudes by computing $\epsilon_{p}$ at selected locations of the complex plane $\lambda$ :

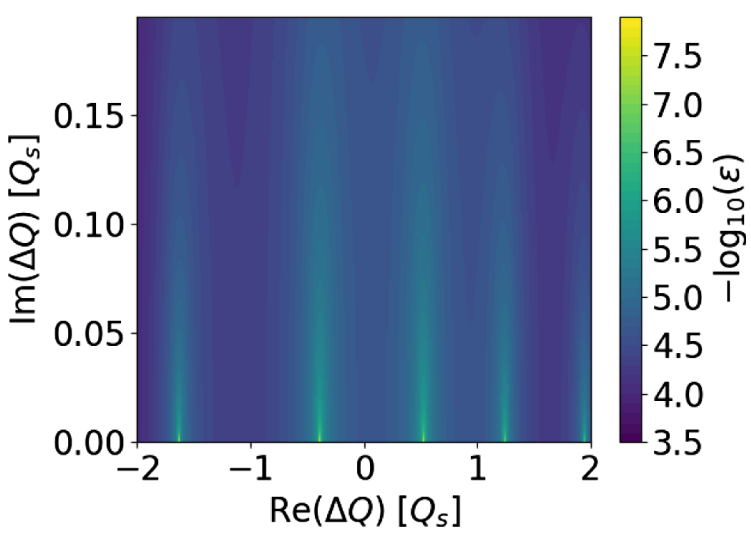

(a)

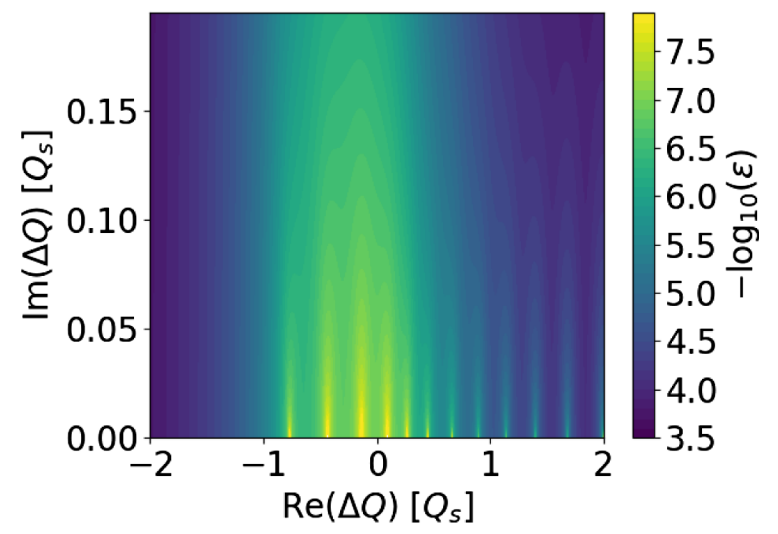

(b)

FIG. 5. Pseudospectrum of the one turn matrix with weak (above) and strong (below) space charge forces, both being above the decoupling of the original TMCI by space charge. The normalized intensity is 20. (a) $\Delta Q_{\mathrm{SC}}=6 Q_{s}$ (b) $\Delta Q_{\mathrm{SC}}=50 Q_{s}$. 


$$
\epsilon_{p}(M, \lambda)=s_{\min }(M-\lambda I)
$$

with $s_{\min }$ denoting the smallest singular value. By comparing the pseudospectrum in the area of the complex plane where the coherent tunes are aggregating, due to the positive mode's tunes approaching asymptotically the one of the mode 0 , we find that the pseudospectrum for a given small perturbation drastically protrudes into the imaginary part of the complex plane when space charge is strong. This observation suggests that the proximity of the mode frequencies results in an increased sensitivity to external perturbations. To quantify this sensitivity with a measurable quantity, we make use of the Kreiss matrix theorem [10] giving in particular a lower bound to the maximum transient amplification that may occur when considering powers of the one turn matrix, i.e., when considering the beam oscillation over several turns:

$$
\sup _{t>0}\left\|M^{t}\right\|>\mathcal{K}(M)
$$

with $t$ the turn counter. In a stable system, the Kreiss constant $\mathcal{K}(M)$ is equal to one, meaning that no exponential growth or transient amplification may be expected. For a system normally unstable, i.e., featuring unstable eigenvalues, the Kreiss constant is infinite. Systems featuring only stable eigenvalues yet having a finite Kreiss constant larger than 1 are subject to transient amplification. Figure 4(b) shows the Kreiss constant obtained for different intensities and space charge strength using

$$
\mathcal{K}(M)=\max _{\Im \lambda>0} \frac{\|\lambda\|-1}{\epsilon_{p}(M, \lambda)} .
$$

We observe that when increasing the strength of space charge forces the Kreiss constant increases significantly. Even for intensities below the TMCI threshold in the absence of space charge the Kreiss constant can be significantly higher than one, such that transient amplification can also be expected. The evolution of the transient behavior with increasing space charge strength resembles the one obtained within the 2PA (Fig. 1). The transient amplification obtained here is compatible with the convective nature of the instabilities obtained within the SSC [6]. In the latter, the transverse oscillation signal was found to be significantly higher for the tail of the bunch with respect to the head. This strong asymmetry between the oscillation amplitude of the head and tail can also be observed with the CMM. Figure 6 illustrates this effect with the superposition of the transverse signals $\tilde{x}(s, t)$ defined as the average transverse position of the bunch multiplied by the line charge density as a function of the longitudinal position $z$, for 20 consecutive turns $t$. The transverse signal is obtained from the expression of the eigenvector corresponding to a given mode $\langle x\rangle_{n, k}$ with its corresponding tune $Q_{n}$ :

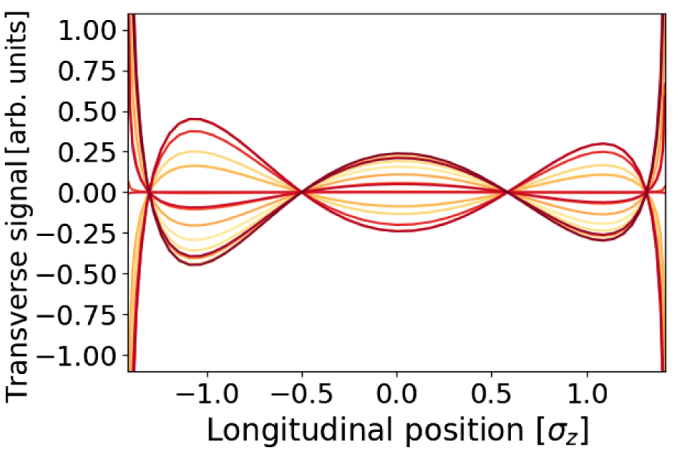

(a)

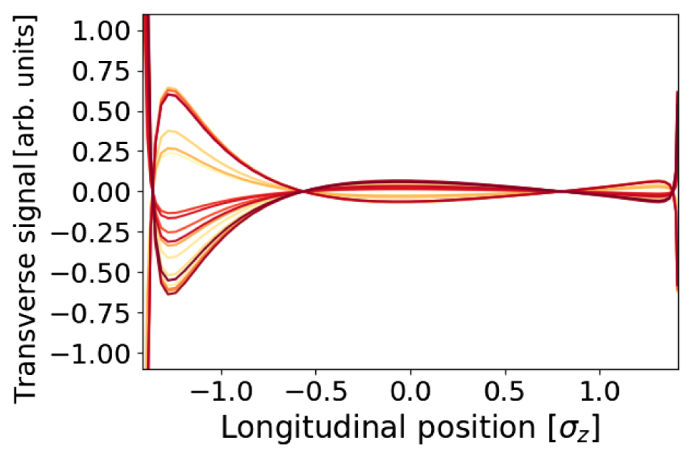

(b)

FIG. 6. Transverse signals obtained for the azimuthal mode 4 for different strengths of the space charge force and for an airbag distribution. The normalized intensity is 20 , i.e., well beyond the TMCI threshold in the absence of space charge forces. The positive longitudinal positions correspond to the head of the bunch, i.e., the part of the beam that arrives first at a given location around the ring. (a) $\Delta Q_{\mathrm{SC}} / Q_{s}=0$ (b) $\Delta Q_{\mathrm{SC}} / Q_{s}=20$.

$$
\begin{aligned}
\tilde{x}(s, t)= & \Re\left[e^{i 2 \pi Q_{n} t} \sum_{k} \iint\langle x\rangle_{n, k} \Psi_{k}^{p}(r, \theta)\right. \\
& \times \hat{\delta}[s-r \cos (\theta)] d r d \theta] .
\end{aligned}
$$

The particular case of an azimuthal mode 4, with unperturbed frequency $Q_{\beta}+4 Q_{s}$ is shown in Fig. 6, recognizable by its four nodes at which no oscillations are observed. Whereas the maximum amplitude of oscillation at the antinodes is similar in the absence of space charge [Fig. 6(a)] there is a strong asymmetry in the first antinode at the head of the bunch $(s \approx 1)$ and the one at the tail $(s \approx-1)$ when space charge forces are strong. It is an important feature of the instability observed experimentally [8]. Nevertheless, while the asymmetry is already present in the model with an airbag distribution, the predicted signals do not resemble the experimental ones. The signals tend to infinity on both edges of the bunch. This is a known issue of the airbag model in a linear $\mathrm{rf}$ system, which is why the ABS is often preferred. Here the airbag distribution in a linear if system only serves as a first step towards a description of a more realistic Gaussian distribution. 


\section{GAUSSIAN DISTRIBUTION}

The description of the longitudinal motion with an airbag distribution entirely neglects the radial degree of freedom. In this case, the unperturbed spectrum features additional degenerate radial modes with frequencies determined solely by the azimuthal mode number. By introducing multiple layers of airbag within the square-well model, it was already shown in [20] that space charge lifts the degeneracy of the radial modes. In particular, the tunes of some radial modes with positive azimuthal number are no longer constrained above the unperturbed transverse tune. This behavior is also observed with the CMM, as illustrated by Fig. 7(a). There, a simplified, semiconverged configuration featuring a sufficient amount of slices but only three rings is shown for reasons that will become obvious later. An important difference with respect to the results obtained with an airbag distribution is the lifting of the degeneracy of the radial modes' frequencies introduced by space charge. This feature is visible at the very left of the plot: the three modes with identical tunes in the absence of space charge forces drift apart as the strength of space charge increases. This results in a much richer spectrum of modes of oscillations and consequently potential for other mode coupling instabilities which are discussed below.

We note that the frequency of the azimuthal and radial mode 0 remains constant at the unperturbed machine tune, the frequency of all other modes decreases with increasing space charge forces at different paces.

Figures 7(b) and 7(c) show an example of the impact of space charge forces in a configuration where, in the absence of space charge, the beams are unstable due to the mode coupling instability driven by the wakefields. Parts 7(b) allow for a better readability of the modes' behavior, yet the low number of rings does not yield fully accurate estimates of the modes' frequencies and growth rate. As with the airbag distribution, we find that the mode coupling instabilities in the absence of space charge forces are decoupled by the increasing space charge force. This is clearly visible by following the behavior of the most unstable mode at the left-hand side of the plot at a tune shift of approximately $-2 Q_{s}$, which is weakened until a full decoupling at a space charge tune shift of $3.4 Q_{s}$. On the other hand, this instability is replaced by another instability at a higher space charge strength, resulting from the coupling of higher order positive azimuthal modes. The behavior of the radial modes involved in this instability is highlighted with black lines. There is an interaction between radial modes of azimuthal number 2 and 4 at a space charge tune shift of $4.7 Q_{s}$. They are highlighted with a dash-dotted and a dashed line respectively. An instability arises where the latter reaches a frequency similar to the one of a radial mode of azimuthal number 5 , marked with a dotted line, at a space charge tune shift of $6.1 Q_{s}$. Since this model has not converged, the behavior cannot be considered accurate, however it helps for a qualitative understanding of the

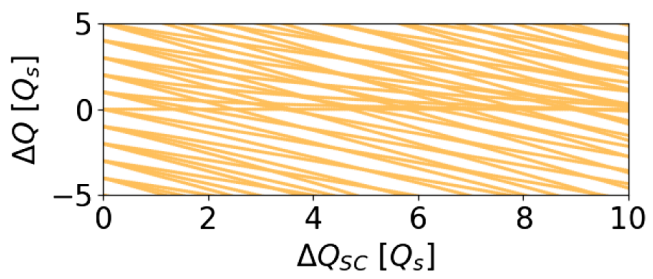

(a)

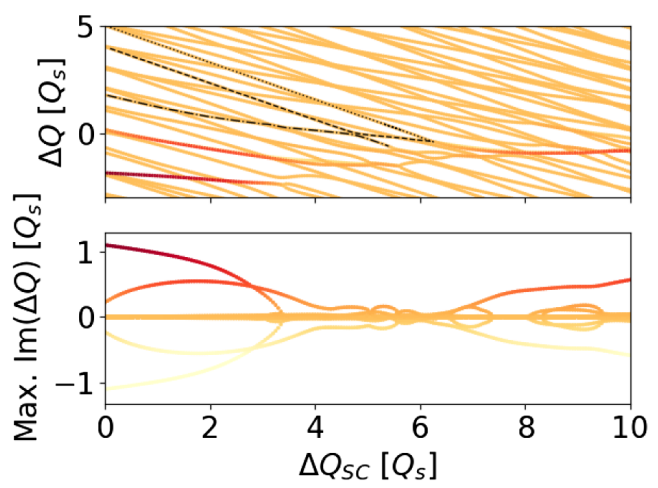

(b)

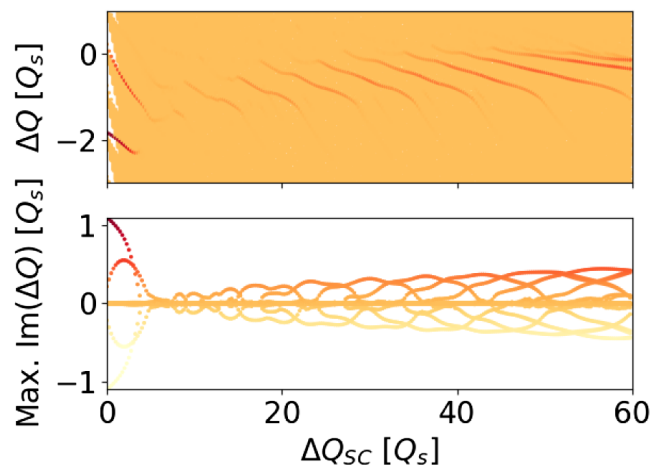

(c)

FIG. 7. Evolution of the complex tune shifts of the coherent modes as a function of the normalized space charge tune shift for a Gaussian distribution. A converged configuration featuring 100 slices and 50 rings is shown on the bottom plot (c), while a semiconverged configuration featuring only three rings is shown on the top (a) and middle plot (b). In the absence of wakefields, in the top plot (a), all tunes are purely real. In the middle plot (b), the behavior of three modes involved in the mode coupling instability are highlighted with black lines. For both plots featuring wakefields (b) and (c), the strength of the wakefields is fixed and corresponds to a normalized intensity of 20 .

behavior observed when considering the converged configuration featuring 50 rings. Indeed, the decoupling of the original mode coupling instabilities in the absence of space charge and the replacement by a succession of mode coupling and decoupling is well visible in Fig. 7(c), however the density of the spectrum prevents a detailed understanding of the role of each mode. The suppression of the original mode coupling instability and the generation of new ones when increasing the space charge strength seem 


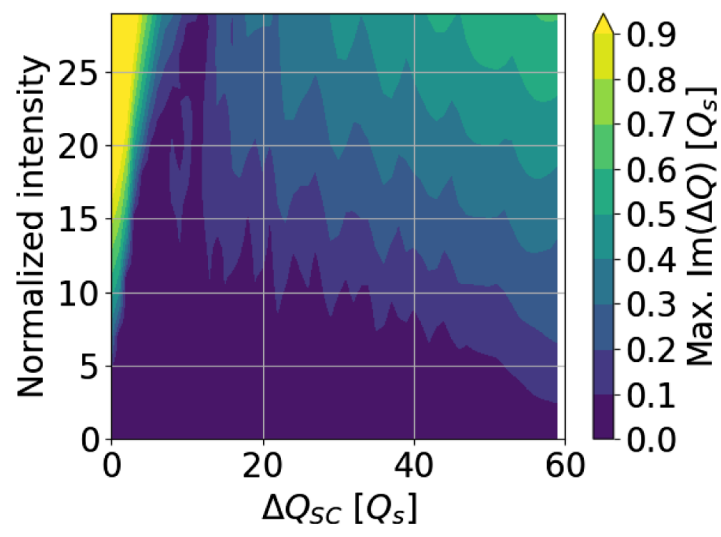

FIG. 8. Maximum imaginary tune shift for a Gaussian distribution obtained with 100 slices and 50 rings for different strengths of the wakefields and the space charge forces.

in agreement with the predictions of the 2PA (Fig. 1). The main difference with 2PA is the width of the mode couplings, which is thin enough in the 2PA to allow intermediate stable configurations. Here we find that the mode couplings overlap with each other, such that there are no intermediate ranges of stability.

The qualitative agreement with the $2 \mathrm{PA}$ approach is also visible in the maximum imaginary part obtained with the CMM on a wider range of intensities shown in Fig. 8. For a given intensity leading to TMCI in the absence of space charge forces, there exists an intermediate strength of the space charge forces that mitigates the original TMCI. Yet, at higher space charge strength, the space charge forces lead to instabilities via the coupling of positive head-tail modes. Compatibly with the 2PA, we find the TMCI threshold vanishes for high space charge strength [7].

As with the airbag distribution, we compute the transverse signal corresponding to the most unstable mode in a configuration without space charge [Fig. 9(a)] and featuring strong space charge, i.e., such that the original instability mechanism is suppressed and replaced by another mode coupling of positive azimuthal modes [Fig. 9(b)]. There are no nodes in both cases. With strong space charge forces, the maximum of the oscillation is displaced towards the tail and the oscillation amplitude is suppressed at the head of the bunch. This asymmetry in the oscillation amplitude of the head and the tail is comparable to the one obtained with the airbag distribution. However here the transverse signals match qualitatively the measured ones [8], suggesting that the model properly describes the instability mechanism observed.

\section{COMPARISON TO MEASUREMENTS AT THE SPS}

In order to validate the new model, we attempt to reproduce the results of an experiment conducted at the SPS and reported in [8]. This experiment was conducted

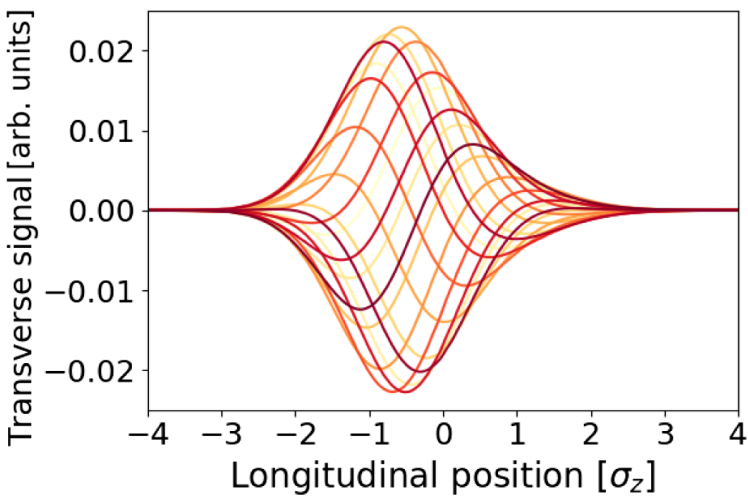

(a)

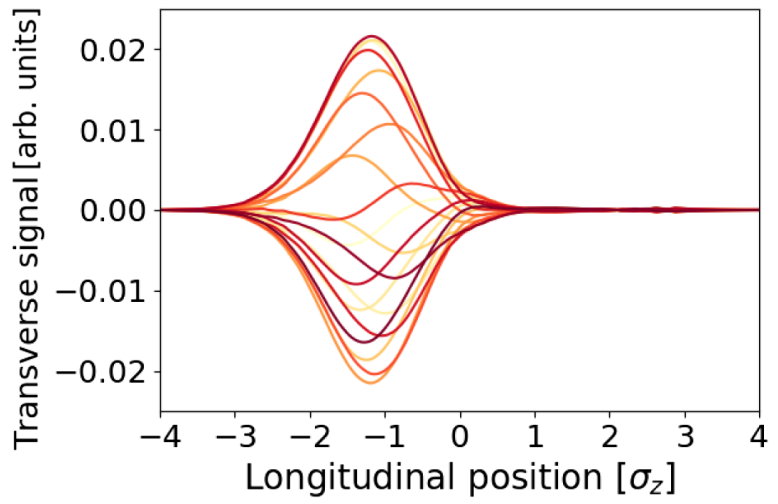

(b)

FIG. 9. Transverse signals obtained for the most unstable mode for different strengths of the space charge force and for a Gaussian distribution. The normalized intensity is 20. The positive longitudinal positions correspond to the head of the bunch, i.e., the part of the beam that arrives first at a given location around the ring. (a) $\Delta Q_{\mathrm{SC}} / Q_{s}=0$ (b) $\Delta Q_{\mathrm{SC}} / Q_{s}=20$.

with two optics, so-called Q26 and Q20, featuring different transition energies. Bunches of various intensities were injected and their stability was evaluated through the losses measured within the first 1000 turns. The space charge tune shift, in units of the synchrotron tune, was kept approximately constant at $28 \pm 2$ with the Q26 optics and $8 \pm 2$ with the Q20 optics.

Here we use the SPS impedance model instead of the broadband resonator model [21]. As shown in [12,13], we observe in Fig. 10 that both the driving and detuning wakefields have an impact on the TMCI. It was checked with the same model that the chromaticity as measured during the experiment has a negligible impact on the growth rate.

With the Q26 optics [Fig. 10(a)], the TMCI threshold without space charge is predicted at an intensity of $1.5 \times 10^{11} \mathrm{p}$, while weak instabilities are expected for intensities between 0.5 and $1 \times 10^{11} \mathrm{p}$. When including space charge forces, the lower end of the error bar is compatible with a TMCI threshold at $0.8 \times 10^{11}$, which is significantly lower than the $1.2 \times 10^{11}$ measured. This 


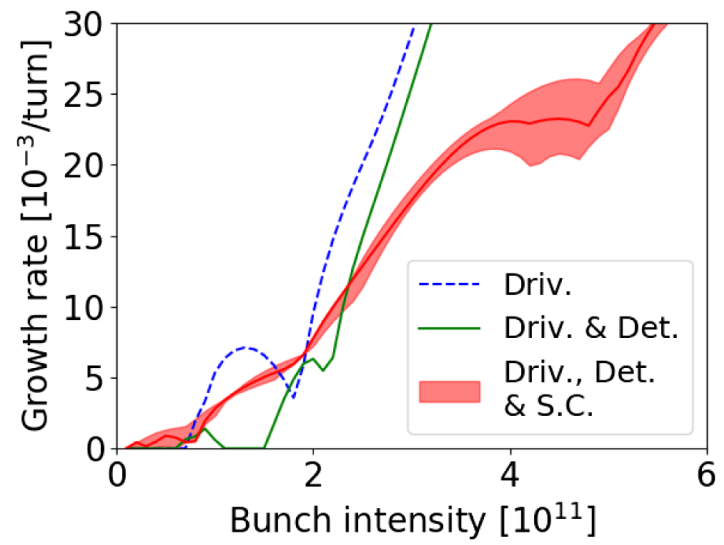

(a)

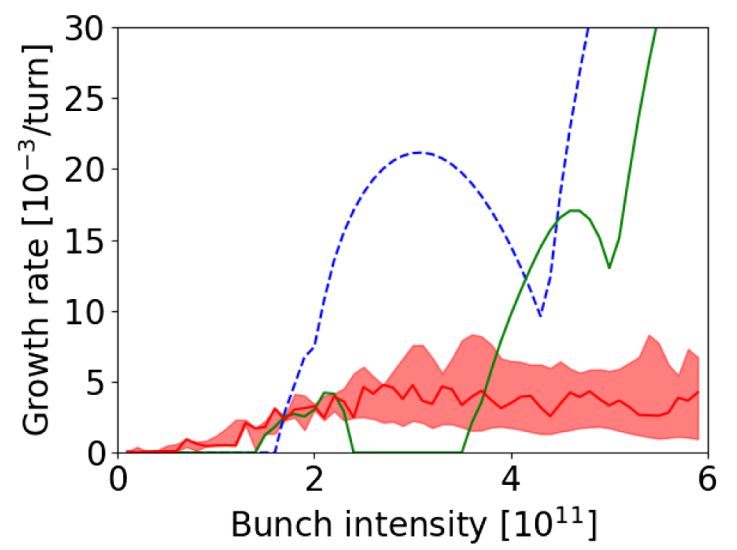

(b)

FIG. 10. Maximum growth rate obtained for a Gaussian distribution in configurations corresponding to an experiment conducted at the SPS with two different optics labeled Q26 (above) and Q20 (below) [8]. The result of the CMM without space charge forces but with either only the detuning wakefields or both the detuning and driving wakefields are shown with blue and green curves respectively. They were obtained with 80 slices and 40 rings. The red lines and shaded areas represent the configuration featuring both driving and detuning wakefields as well as space charge forces, with a range given by the uncertainty on the transverse and longitudinal emittances. They were obtained with 140 slices and 60 rings. (a) Q26 (b) Q20.

important inaccuracy in the model may be attributed to the linearization of the space charge forces; Landau damping is thus entirely neglected. Nevertheless, the predicted growth rates are in good agreement for bunches of sufficiently high intensity, in the regime of fast losses. Indeed the growth rate was measured at approximatively $6 \times 10^{-3} /$ turn for an intensity of $1.5 \times 10^{11}$. One may suppose that the linear model is more accurate in this regime because Landau damping is lost.

With the Q20 optics [Fig. 10(b)] and without space charge, instabilities with rise times shorter than 1000 turns are already expected at intensities between 1.4 and $2.4 \times 10^{11} \mathrm{p}$. Above these intensities, the beam is expected to be stable until an intensity of $3.5 \times 10^{11} \mathrm{p}$. When including space charge, we observe instabilities for all intensities above approximately $10^{11}$. In this regime of rather weak space charge forces, the uncertainty on the beam parameters plays a significant role as it leads to larger error bars, with respect to the regime of strong space charge force with the Q26 optics. This feature is compatible with the experimental data. Indeed, strong shot-to-shot variations were observed in the beam stability when operating with the Q20 optics, which were not observed with the Q26 optics. This behavior may be attributed to unmeasured shot-to-shot variations of the transverse and longitudinal emittances, having a marginal impact on the beam stability with the Q26 optics due to the strong space charge forces, but having a significant impact with lower space charge forces when operating with the Q20 optics.

Experimentally, only weak instabilities were observed with intensities above $1.5 \times 10^{11} \mathrm{p}$, strong instabilities with rise time shorter than 1000 turns occurred only above $3.5 \times 10^{11} \mathrm{p}$. Again these features cannot be explained within the CMM, likely due to the absence of Landau damping in the model.

The introduction of space charge forces does not seem to provide a significant improvement in the prediction of the instability growth rate in the SPS with respect to the same model without space charge, featuring only the driving and detuning wakefields. In fact, it was already observed in [8] that macroparticle simulations featuring only these ingredients, i.e., excluding space charge forces, yielded predictions in reasonable agreement with experimental data (except for the strong shot-to-shot variations with the Q20 optics). Nevertheless, the CMM model including space charge forces shows that growth rates compatible with experimental data can be obtained with a linearized model, yet the introduction of the nonlinearities seem necessary for a more accurate description.

\section{CONCLUSION}

Several results obtained with simplified models featuring different types of approximation were recovered with a new dynamical model of beam instabilities in synchrotrons featuring wakefields and space charge forces. First, the behavior of the frequencies of the coherent modes under the influence of space charge forces, the suppression of the TMCI and the existence of significant transient growth in spite of the lack of unstable eigenvalues were recovered within the airbag model. In particular, the transient growth was quantified by means of the Kreiss constant of the one turn matrix obtained with the CMM. Second, the reduction of the frequency of some radial modes featuring positive azimuthal mode numbers below the unperturbed tune caused by space charge forces was recovered [20]. With the CMM we find that these modes can couple due to the space charge forces and thus result in instabilities at high space charge strength, as opposed to the prediction of the 
airbag model. This behavior, i.e., the mitigation of the original TMCI and the generation of multiple mode couplings with increasing space charge forces, is compatible with the one obtained with the $2 \mathrm{PA}$ prediction. On the other hand, the coupling of the radial modes seems in contradiction with the results in [5]. Nevertheless, the model used in the latter was obtained with the SSC approximation, which is not met at the edge of a longitudinal Gaussian distribution. Since the CMM is not based on such an approximation, the difference can likely be attributed to that.

We note that recently the existence of normal instabilities, i.e., described by unstable eigenvalues, in the presence of wakefields and strong space charge forces was also obtained using Vlasov perturbation theory without the SSC [22]. It is suggested that the transverse variation of the space charge tune shift plays an important role in the generation of the instability, following a mechanism described in [23]. Since the space charge forces are linearized with respect to the transverse position in the CMM, transverse variations of the tune shift cannot impact the instability mechanism described in this paper, consequently they are of different nature.

The growth rates and transverse signals measured in an experiment at the SPS were found in qualitative agreement with the predictions of the CMM, yet some configurations were found more stable experimentally than theoretically. This difference may be attributed to the absence of modeling of Landau damping in the linearized model of space charge forces implemented in the CMM.

\section{ACKNOWLEDGMENTS}

We would like to express our gratitude to $\mathrm{H}$. Bartosik and C. Zannini for their help in the comparison with past experimental data, as well as to G. Arduini, Y. Papaphilippou and R. Jones for proofreading the manuscript.

\section{APPENDIX A: CONVERGENCE}

The variation of the main observable obtained from the $\mathrm{CMM}$, i.e., the maximum growth rate, is shown for a given configuration and for different numbers of slices and rings in Fig. 11. The result does not vary significantly for a number of slices higher than 60 and for more than 2000 discretized elements and thus is considered converged. These requirements increase with the space charge strength as higher order positive head-tail modes are shifted down in frequency and lead to mode coupling instabilities. The number of rings has to be increased accordingly to allow for a sufficiently accurate description of the forces. As a result, the CMM becomes numerically challenging for high space charge strength. Nevertheless, 100 slices and 50 rings were found sufficient for a wide range of realistic parameters. This numerical configuration leads a matrix size of $10^{4} \times 10^{4}$ yielding acceptable computing time.

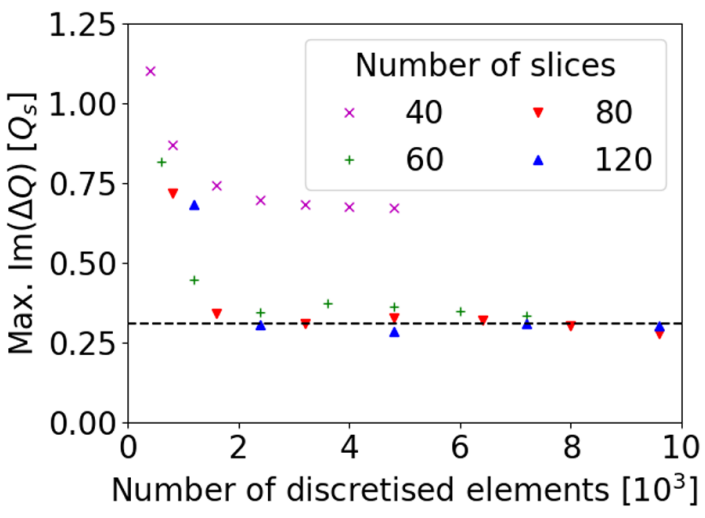

FIG. 11. Maximum imaginary tune shift obtained for a Gaussian distribution, a normalized space charge tune shift of 30 , a normalized intensity of 20 for various discretizations of the longitudinal phase space. The number of discretized elements is quoted on the $\mathrm{x}$ axis as it relates directly to the computational need, it is simply given by $N_{s} \cdot N_{r}$. A converged value is highlighted with a dashed black line.

\section{APPENDIX B: TRANSIENT AMPLIFICATION}

The Kreiss constant allows to quantify the transient behavior of a dynamical system, yet the correspondence to physical observables is not trivial. In this section we seek to illustrate this aspect with a concrete example, thus also clarifying the link with the convective behavior described in [6].

Figure 12 shows an example of transient behavior observed in the presence of wakefields and space charge

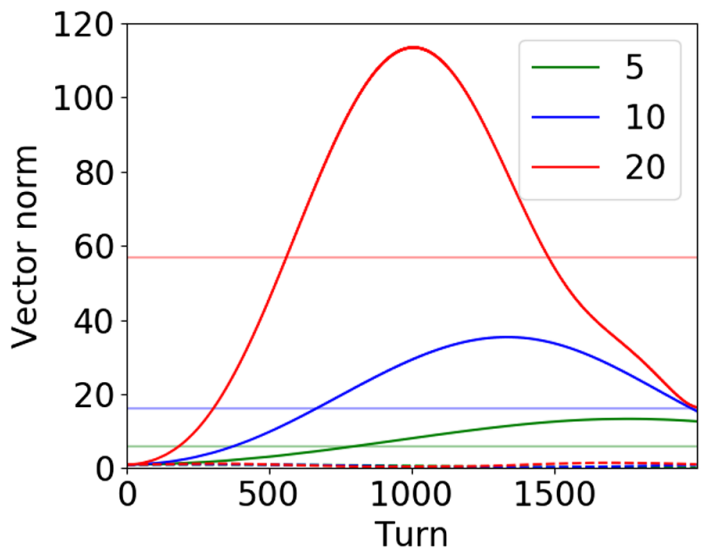

FIG. 12. Evolution of the norm of a vector associated with two different initial perturbations under multiplications by the one turn matrix $M$. The one turn matrix corresponds to a normalized intensity of 20, the space charge tune shift is 5, 10 and 20 for the green, blue and red curves respectively. The airbag model was considered with 100 slices. The solid and dashed lines correspond to two different initial perturbations, corresponding to a displacement of the head and tail of the bunch respectively. The shaded curves show the Kreiss constant associated with the corresponding one turn matrix. 


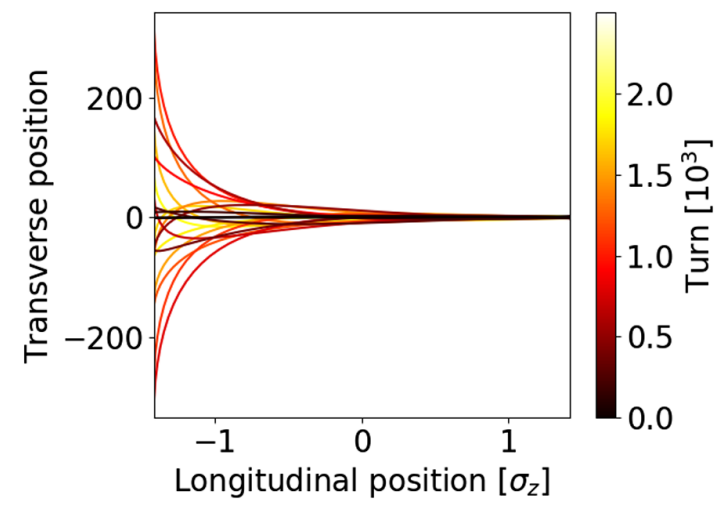

FIG. 13. Evolution of an initial perturbation, corresponding to a displacement of the head of the bunch, under multiplications by the one turn matrix corresponding to a normalized intensity of 20 and a space charge tune shift of 20 . The airbag model was considered with 100 slices. The transverse position is normalized to the maximum offset at the head of the bunch in the initial perturbation.

in a regime where all eigenvalues are stable, yet the Kreiss constant is larger than 1 . Two initial perturbations were considered. The first one features a displacement of the head of the bunch, with a position that is set to 0 for the trailing half of the bunch and an offset proportional to the longitudinal position for the leading half (solid lines). The second features a similar perturbation of the trailing half without perturbation of the leading half (dashed lines). In both cases, the vector representing the initial perturbation is normalized to 1 . For the configuration where the head of the bunch is perturbed, the norm of the vector increases until a maximum before decaying again, thus representing a transient amplification of the initial perturbation. On the other hand, the perturbation of the tail of the bunch is barely amplified, showing that the amplification of the initial perturbation exhibits a strong dependence on the initial conditions. Thus, this measure can hardly be used to quantify and compare different configurations, whereas the Kreiss constant allows for a quantitative measure of the potential for transient amplification of a given matrix. In these examples, the maximum of the amplification is indeed of the order of the Kreiss constant, represented with shaded lines on Fig. 12. We recall that the Kreiss constant provides a lower bound for the maximum transient amplification. It was therefore expected that the maximum reached in these examples is higher than the Kreiss constant.

The main concern for accelerators is that transient amplifications may become sufficiently large that beam losses or emittance growth occur. The Kreiss matrix theorem is of limited value to obtain accurate estimations of these quantities, mostly because the upper bound is often much larger than the values actually achieved [19] and the lower bound is not sufficient to fully characterize the potential detrimental effects on the beam quality.
Figure 13 shows the expected beam position along the bunch after multiple turns for the configuration discussed above, with an initial perturbation of the head of the bunch. This initial perturbation leads to an amplification of the motion towards the tail of the bunch, whereas the motion at the head is not amplified. This amplification along the bunch is comparable to the convective behavior quoted in [6]. We note that, here, the amplification is bound in amplitude. It occurs in the first thousand turns (black to light red lines) before reaching a maximum and decaying again (light red to yellow lines), compatibly with the behavior observed in Fig. 12 (solid blue line). While transient, the oscillation amplitude at the tail of the bunch reaches more than 200 times the initial perturbation of the head, which could indeed result in beam losses or emittance growth.

[1] M. Blaskiewicz, Phys. Rev. ST Accel. Beams 1, 044201 (1998).

[2] V. Balbekov, Phys. Rev. ST Accel. Beams 15, 054403 (2012).

[3] A. Burov, Phys. Rev. ST Accel. Beams 12, 044202 (2009).

[4] T. Zolkin, A. Burov, and B. Pandey, Phys. Rev. Accel. Beams 21, 104201 (2018).

[5] V. Balbekov, Phys. Rev. Accel. Beams 22, 044401 (2019).

[6] A. Burov, Phys. Rev. Accel. Beams 22, 034202 (2019).

[7] Y. H. Chin, A. W. Chao, and M. M. Blaskiewicz, Phys. Rev. Accel. Beams 19, 014201 (2016).

[8] H. Bartosik, Beam dynamics and optics studies for the LHC injectors upgrade, Ph.D. thesis, Technische Unisersität Wien, 2013.

[9] V. Danilov and E. Perevedentsev, Nucl. Instrum. Methods Phys. Res., Sect. A 391, 77 (1997).

[10] H.-O. Kreiss, BIT Numerical Mathematics 2, 153 (1962).

[11] B. Zotter and S. Kheifets, Impedances and Wakes in HighEnergy Particle Accelerators (World Scientific, Singapore, 1998).

[12] A. Burov and V. Danilov, Phys. Rev. Lett. 82, 2286 (1999).

[13] E. Métral, X. Buffat, and G. Rumolo, Transverse modecoupling instability in the presence of detuning impedance, Technical Report No. ACC-NOTE-2020-0019, CERN, Geneva, Switzerland, 2020.

[14] E. A. Perevedentsev and A. A. Valishev, Phys. Rev. ST Accel. Beams 4, 024403 (2001).

[15] S. White, X. Buffat, N. Mounet, and T. Pieloni, Phys. Rev. ST Accel. Beams 17, 041002 (2014).

[16] M. Schenk, X. Buffat, K. Li, and A. Maillard, Phys. Rev. Accel. Beams 21, 084402 (2018).

[17] X. Buffat, Transverse beams stability studies at the Large Hadron Collider, Ph.D. thesis, École Polytechnique Fédérale de Lausanne, 2015.

[18] K. Schindl, in CAS-CERN Accelerator School: Intermediate Course on Accelerator Physics, edited by D. Brandt (CERN, Geneva, Switzerland, 2006), pp. 305-320.

[19] L. N. Trefethen and M. Embree, Spectra and Pseudospectra: The Behavior of Nonnormal Matrices and Operators (Princeton University Press, Princeton, 2005). 
[20] V. Balbekov, Phys. Rev. Accel. Beams 20, 034401 (2017).

[21] C. Zannini, Electromagnetic simulation of CERN accelerator components and experimental applications, Ph.D. thesis, École Polytechnique Fédérale de Lausanne, 2013.
[22] Y. Alexahin, in Proceedings of the ICFA Mini-Workshop on Mitigation of Coherent Instabilities in Particle Accelerators MCBI2019, edited by E. Métral, T. Pieloni, and G. Rumolo (Zermatt, Switzerland, 2019).

[23] A. Burov, Core-halo collective instabilities, arXiv:1808 .08498 . 